\title{
Search for chargino pair production in scenarios with gravitino LSP and stau NLSP at $\sqrt{\mathrm{s}} \sim 183 \mathrm{GeV}$ at LEP
}

DELPHI Collaboration

\begin{abstract}
Promptly decaying lightest charginos were searched for in the context of scenarios with gravitino LSP. It was assumed that the stau is the next to lightest supersymmetric particle (NLSP). Data collected with the DELPHI detector at a centre-of-mass energy near $183 \mathrm{GeV}$ were analysed combining the methods developed in previous searches. No evidence for the production of these particles was found. Hence, limits were derived at $95 \%$ confidence level. The mass of charginos was found to be greater than $85.5 \mathrm{GeV} / c^{2}$ for $m_{\tilde{\chi}_{1}^{+}}-m_{\tilde{\tau}_{1}} \geq 0.3 \mathrm{GeV} / c^{2}$, independently of the mass of the gravitino.
\end{abstract}


P.Abreu ${ }^{22}$, W.Adam ${ }^{52}, \quad$ T.Adye ${ }^{38}, \quad$ P.Adzic $^{12}, \quad$ Z.Albrecht ${ }^{18}, \quad$ T.Alderweireld ${ }^{2}$, G.D.Alekseev ${ }^{17}, \quad$ R.Alemany ${ }^{51}$, T.Allmendinger ${ }^{18}$, P.P.Allport ${ }^{23}$, S.Almehed ${ }^{25}$, U.Amaldi ${ }^{9}$, N.Amapane ${ }^{47}$, S.Amato ${ }^{49}$, E.G.Anassontzis ${ }^{3}$, P.Andersson ${ }^{46}$, A.Andreazza $^{9}$, S.Andringa ${ }^{22}$, P.Antilogus ${ }^{26}$, W-D.Apel ${ }^{18}$, Y.Arnoud ${ }^{9}$, B.Åsman ${ }^{46}$, J-E.Augustinn ${ }^{26}$, A.Augustinus ${ }^{9}$, P.Baillon $^{9}$, P.Bambade ${ }^{20}$, F.Barao ${ }^{22}$, G.Barbiellini ${ }^{48}$, R.Barbier $^{26}$, D.Y.Bardin ${ }^{17}$, G.Barker ${ }^{18}$, A.Baroncelli ${ }^{40}$, M.Battaglia ${ }^{16}$, M.Baubillier ${ }^{24}$, K-H.Becks ${ }^{54}$, M.Begalli ${ }^{6}$, A.Behrmann ${ }^{54}$, P.Beilliere ${ }^{8}$, Yu.Belokopytov ${ }^{9}$, K.Belous ${ }^{44}$, N.C.Benekos ${ }^{33}$, A.C.Benvenuti ${ }^{5}$, C.Berat ${ }^{15}$, M.Berggren ${ }^{26}$, D.Bertini ${ }^{26}$, D.Bertrand ${ }^{2}$, M.Besancon ${ }^{41}$, M.Bigi $^{47}$, M.S.Bilenky ${ }^{17}$, M-A.Bizouard ${ }^{20}$, D.Bloch ${ }^{10}$, H.M.Blom ${ }^{32}$, M.Bonesini $^{29}$, W.Bonivento ${ }^{28}$, M.Boonekamp $^{41}$, P.S.L.Booth ${ }^{23}$, A.W.Borgland ${ }^{4}$, G.Borisov ${ }^{20}$, C.Bosio ${ }^{43}$, O.Botner ${ }^{50}$, E.Boudinov ${ }^{32}$, B.Bouquet ${ }^{20}$, C.Bourdarios ${ }^{20}$, T.J.V.Bowcock ${ }^{23}$, I.Boyko $^{17}$, I.Bozovic $^{12}$, M.Bozzo ${ }^{14}$, M.Bracko ${ }^{45}$, P.Branchini ${ }^{40}$, R.A.Brenner ${ }^{50}$, P.Bruckman ${ }^{9}$, J-M.Brunet ${ }^{8}$, L.Bugge ${ }^{34}$, T.Buran $^{34}$, B.Buschbeck ${ }^{52}$, P.Buschmann ${ }^{54}$, S.Cabrera ${ }^{51}$, M.Caccia ${ }^{28}$, M.Calvi $^{29}$, T.Camporesi ${ }^{9}$, V.Canale ${ }^{39}$, F.Carena ${ }^{9}$, L.Carroll ${ }^{23}$, C.Caso ${ }^{14}$, M.V.Castillo Gimenez ${ }^{51}$, A.Cattai ${ }^{9}$, F.R.Cavallo ${ }^{5}$, V.Chabaud ${ }^{9}$, M.Chapkin ${ }^{44}$, Ph.Charpentier $^{9}$, L.Chaussard $^{26}$, P.Checchia ${ }^{37}$, G.A.Chelkov ${ }^{17}$, R.Chierici ${ }^{47}$, P.Chliapnikov ${ }^{44}$, P.Chochula ${ }^{7}$, V.Chorowicz ${ }^{26}$, J.Chudoba ${ }^{31}$, K.Cieslik ${ }^{19}$, P.Collins ${ }^{9}$, R.Contri ${ }^{14}$, E.Cortina ${ }^{51}$, G.Cosme ${ }^{20}$, F.Cossutti ${ }^{9}$, H.B.Crawley ${ }^{1}$, D.Crennell ${ }^{38}$, S.Crepe ${ }^{15}$, G.Crosetti $^{14}$, J.Cuevas Maestro ${ }^{35}$, S.Czellar ${ }^{16}$, M.Davenport ${ }^{9}$, W.Da Silva ${ }^{24}$, A.Deghorain ${ }^{2}$, G.Della Ricca ${ }^{48}$, P.Delpierre ${ }^{27}$, N.Demaria ${ }^{9}$, A.De Angelis ${ }^{9}$, W.De Boer ${ }^{18}$, C.De Clercq ${ }^{2}$, B.De Lotto ${ }^{48}$, A.De Min ${ }^{37}$, L.De Paula ${ }^{49}$, H.Dijkstra ${ }^{9}$, L.Di Ciaccio ${ }^{39,9}$, J.Dolbeau ${ }^{8}$, K.Doroba ${ }^{53}$, M.Dracos ${ }^{10}$, J.Drees ${ }^{54}$, M.Dris ${ }^{33}$, A.Duperrin ${ }^{26}$, J-D.Durand ${ }^{9}$, G.Eigen $^{4}$, T.Ekelof ${ }^{50}$, G.Ekspong ${ }^{46}$, M.Ellert ${ }^{50}$, M.Elsing ${ }^{9}$, J-P.Engel ${ }^{10}$, M.Espirito Santo ${ }^{22}$, G.Fanourakis ${ }^{12}$, D.Fassouliotis ${ }^{12}$, J.Fayot ${ }^{24}$, M.Feindt ${ }^{18}$, P.Ferrari ${ }^{28}$, A.Ferrer ${ }^{51}$, E.Ferrer-Ribas ${ }^{20}$, F.Ferro ${ }^{14}$, S.Fichet ${ }^{24}$, A.Firestone ${ }^{1}$, U.Flagmeyer $^{54}$, H.Foeth ${ }^{9}$, E.Fokitis ${ }^{33}$, F.Fontanelli ${ }^{14}$, B.Franek ${ }^{38}$, A.G.Frodesen ${ }^{4}$, R.Fruhwirth ${ }^{52}$, F.Fulda-Quenzer ${ }^{20}$, J.Fuster $^{51}$, A.Galloni ${ }^{23}$, D.Gamba ${ }^{47}$, S.Gamblin ${ }^{20}$, M.Gandelman $^{49}$, C.Garcia ${ }^{51}$, C.Gaspar ${ }^{9}$, M.Gaspar $^{49}$, U.Gasparini $^{37}$, Ph.Gavillet $^{9}$, E.N.Gazis ${ }^{33}$, D.Gele ${ }^{10}$, N.Ghodbane ${ }^{26}$, I.Gil ${ }^{51}$, F.Glege ${ }^{54}$, R.Gokieli ${ }^{9,53}$, B.Golob ${ }^{45}$, G.Gomez-Ceballos ${ }^{42}$, P.Goncalves ${ }^{22}$, I.Gonzalez Caballero ${ }^{42}$, G.Gopal ${ }^{38}$, L.Gorn ${ }^{1}$, Yu.Gouz ${ }^{44}$, V.Gracco ${ }^{14}$, J.Grahl ${ }^{1}$, E.Graziani ${ }^{40}$, P.Gris ${ }^{41}$, G.Grosdidier ${ }^{20}$, K.Grzelak ${ }^{53}$, J.Guy ${ }^{38}$, F.Hahn ${ }^{9}$, S.Hahn ${ }^{54}$, S.Haider ${ }^{9}$, A.Hallgren ${ }^{50}$, K.Hamacher ${ }^{54}$, J.Hansen ${ }^{34}$, F.J.Harris ${ }^{36}$, V.Hedberg ${ }^{25}$, S.Heising ${ }^{18}$, J.J.Hernandez ${ }^{51}$, P.Herquet ${ }^{2}$, H.Herr ${ }^{9}$, T.L.Hessing ${ }^{36}$, J.-M.Heuser ${ }^{54}$, E.Higon ${ }^{51}$, S-O.Holmgren ${ }^{46}$, P.J.Holt ${ }^{36}$, S.Hoorelbeke ${ }^{2}, \quad$ M.Houlden ${ }^{23}$, J.Hrubec ${ }^{52}$, M.Huber $^{18}$, K.Huet ${ }^{2}$, G.J.Hughes ${ }^{23}$, K.Hultqvist ${ }^{46}$, J.N.Jackson ${ }^{23}$, R.Jacobsson ${ }^{9}$, P.Jalocha ${ }^{19}$, R.Janik ${ }^{7}, \quad$ Ch.Jarlskog ${ }^{25}$, G.Jarlskog ${ }^{25}, \quad$ P.Jarry ${ }^{41}$, B.Jean-Marie ${ }^{20}$, D.Jeans ${ }^{36}$, E.K.Johansson ${ }^{46}$, P.Jonsson ${ }^{26}$, C.Joram ${ }^{9}$, P.Juillot ${ }^{10}$, L.Jungermann ${ }^{18}$, F.Kapusta ${ }^{24}$, K.Karafasoulis $^{12}$, S.Katsanevas ${ }^{26}$, E.C.Katsoufis ${ }^{33}$, R.Keranen ${ }^{18}$, G.Kernel ${ }^{45}$, B.P.Kersevan ${ }^{45}$, B.A.Khomenko ${ }^{17}$, N.N.Khovanski ${ }^{17}$, A.Kiiskinen ${ }^{16}$, B.King ${ }^{23}$, A.Kinvig ${ }^{23}$, N.J.Kjaer ${ }^{32}$, O.Klapp ${ }^{54}$, H.Klein ${ }^{9}$, P.Kluit ${ }^{32}$, P.Kokkinias ${ }^{12}$, M.Koratzinos $^{9}$, V.Kostioukhine ${ }^{44}$, C.Kourkoumelis ${ }^{3}$, O.Kouznetsov ${ }^{41}$, M.Krammer ${ }^{52}$, E.Kriznic ${ }^{45}$, Z.Krumstein ${ }^{17}$, P.Kubinec $^{7}$, J.Kurowska ${ }^{53}$, K.Kurvinen ${ }^{16}$, J.W.Lamsa ${ }^{1}$, D.W.Lane ${ }^{1}$, V.Lapin ${ }^{44}$, J-P.Laugier ${ }^{41}$, R.Lauhakangas ${ }^{16}$, G.Leder $^{52}$, F.Ledroit ${ }^{15}$, V.Lefebure ${ }^{2}$, L.Leinonen ${ }^{46}$, A.Leisos ${ }^{12}$, R.Leitner ${ }^{31}$, G.Lenzen ${ }^{54}$, V.Lepeltier ${ }^{20}$, T.Lesiak ${ }^{19}$, M.Lethuillier ${ }^{41}$, J.Libby ${ }^{36}$, W.Liebig ${ }^{54}$, D.Liko ${ }^{9}$, A.Lipniacka ${ }^{46}$, I.Lippi ${ }^{37}$, B.Loerstad ${ }^{25}$, J.G.Loken ${ }^{36}$, J.H.Lopes ${ }^{49}$, J.M.Lopez ${ }^{42}$, R.Lopez-Fernandez ${ }^{15}$, D.Loukas ${ }^{12}$, P.Lutz ${ }^{41}$, L.Lyons ${ }^{36}$, J.MacNaughton ${ }^{52}$, J.R.Mahon ${ }^{6}$, A.Maio ${ }^{22}$, A.Malek $^{54}$, T.G.M.Malmgren ${ }^{46}$, S.Maltezos ${ }^{33}$, V.Malychev ${ }^{17}$, F.Mandl ${ }^{52}$, J.Marco ${ }^{42}$, R.Marco ${ }^{42}$, B.Marechal ${ }^{49}$, M.Margoni $^{37}$, J-C.Marin ${ }^{9}$, C.Mariotti ${ }^{9}$, A.Markou ${ }^{12}$, C.Martinez-Rivero ${ }^{20}$, F.Martinez-Vidal ${ }^{51}$, S.Marti i Garcia ${ }^{9}$, J.Masik $^{13}$, N.Mastroyiannopoulos ${ }^{12}$, F.Matorras ${ }^{42}$, C.Matteuzzi ${ }^{29}$, G.Matthiae ${ }^{39}$, F.Mazzucato ${ }^{37}$, M.Mazzucato ${ }^{37}$, M.Mc Cubbin $^{23}$, R.Mc Kay ${ }^{1}$, R.Mc Nulty ${ }^{23}$, G.Mc Pherson ${ }^{23}$, C.Meroni ${ }^{28}$, W.T.Meyer ${ }^{1}$, E.Migliore ${ }^{9}$, L.Mirabito ${ }^{26}$, W.A.Mitaroff ${ }^{52}$, U.Mjoernmark ${ }^{25}$, T.Moa ${ }^{46}$, M.Moch $^{18}$, R.Moeller ${ }^{30}$, K.Moenig ${ }^{9,11}$, M.R.Monge ${ }^{14}$, X.Moreau ${ }^{24}$, P.Morettini $^{14}$, G.Morton ${ }^{36}$, U.Mueller ${ }^{54}$, K.Muenich ${ }^{54}$, M.Mulders ${ }^{32}$, C.Mulet-Marquis ${ }^{15}$, R.Muresan ${ }^{25}$, W.J.Murray ${ }^{38}$, B.Muryn $^{15,19}$, G.Myatt ${ }^{36}$, T.Myklebust ${ }^{34}$, F.Naraghi ${ }^{15}$, M.Nassiakou ${ }^{12}$, F.L.Navarria ${ }^{5}$, S.Navas ${ }^{51}$, K.Nawrocki ${ }^{53}$, P.Negri $^{29}$, N.Neufeld ${ }^{9}$, R.Nicolaidou ${ }^{41}$, B.S.Nielsen ${ }^{30}$, P.Niezurawski ${ }^{53}$, M.Nikolenko ${ }^{10,17}$, V.Nomokonov ${ }^{16}$, A.Nygren ${ }^{25}$, V.Obraztsov ${ }^{44}$, A.G.Olshevski ${ }^{17}$, A.Onofre ${ }^{22}$, R.Orava ${ }^{16}$, G.Orazi ${ }^{10}$, K.Osterberg ${ }^{16}$, A.Ouraou ${ }^{41}$, M.Paganoni ${ }^{29}$, S.Paiano $^{5}$, R.Pain ${ }^{24}$, R.Paiva ${ }^{22}$, J.Palacios ${ }^{36}$, H.Palka ${ }^{19}$, Th.D.Papadopoulou ${ }^{33,9}$, K.Papageorgiou ${ }^{12}$, L.Pape ${ }^{9}$, C.Parkes ${ }^{9}$, F.Parodi ${ }^{14}$, U.Parzefall ${ }^{23}$, A.Passeri ${ }^{40}$, O.Passon ${ }^{54}$, T.Pavel ${ }^{25}$, M.Pegoraro ${ }^{37}$, L.Peralta ${ }^{22}$, M.Pernicka ${ }^{52}$, A.Perrotta ${ }^{5}$, C.Petridou $^{48}$, A.Petrolini ${ }^{14}$, H.T.Phillips ${ }^{38}$, F.Pierre ${ }^{41}$, M.Pimenta ${ }^{22}$, E.Piotto ${ }^{28}$, T.Podobnik ${ }^{45}$, M.E.Pol ${ }^{6}$, G.Polok ${ }^{19}$, P.Poropat $^{48}$, V.Pozdniakov ${ }^{17}$, P.Privitera ${ }^{39}$, N.Pukhaeva ${ }^{17}$, A.Pullia ${ }^{29}$, D.Radojicic ${ }^{36}$, S.Ragazzi ${ }^{29}$, H.Rahmani ${ }^{33}$, J.Rames $^{13}$, P.N.Ratoff ${ }^{21}$, A.L.Read ${ }^{34}$, P.Rebecchi ${ }^{9}$, N.G.Redaelli ${ }^{28}$, M.Regler $^{52}$, J.Rehn ${ }^{18}$, D.Reid ${ }^{32}$, R.Reinhardt $^{54}$, P.B.Renton ${ }^{36}$, L.K.Resvanis ${ }^{3}$, F.Richard ${ }^{20}$, J.Ridky ${ }^{13}$, G.Rinaudo ${ }^{47}, \quad$ O.Rohne ${ }^{34}, \quad$ A.Romero ${ }^{47}, \quad$ P.Ronchese $^{37}$, E.I.Rosenberg ${ }^{1}$, P.Rosinsky ${ }^{7}$, P.Roudeau ${ }^{20}$, T.Rovelli ${ }^{5}$, Ch.Royon ${ }^{41}$, V.Ruhlmann-Kleider ${ }^{41}$, A.Ruiz ${ }^{42}$, H.Saarikko ${ }^{16}$, Y.Sacquin ${ }^{41}$, A.Sadovsky ${ }^{17}$, G.Sajot ${ }^{15}$, J.Salt ${ }^{51}$, D.Sampsonidis ${ }^{12}$, M.Sannino ${ }^{14}$, Ph.Schwemling ${ }^{24}$, B.Schwering ${ }^{54}$, U.Schwickerath ${ }^{18}$, F.Scuri ${ }^{48}$, P.Seager ${ }^{21}$, Y.Sedykh ${ }^{17}$, A.M.Segar $^{36}$, N.Seibert ${ }^{18}$, R.Sekulin ${ }^{38}$, R.C.Shellard ${ }^{6}$, M.Siebel $^{54}$, L.Simard ${ }^{41}$, F.Simonetto ${ }^{37}$, A.N.Sisakian ${ }^{17}$, G.Smadja ${ }^{26}$, O.Smirnova ${ }^{25}$, G.R.Smith ${ }^{38}$, A.Sokolov ${ }^{44}$, O.Solovianov ${ }^{44}$, A.Sopczak $^{18}$, R.Sosnowski ${ }^{53}$, T.Spassov ${ }^{22}$, E.Spiriti ${ }^{40}$, S.Squarcia ${ }^{14}$, C.Stanescu ${ }^{40}, \quad$ S.Stanic $^{45}, \quad$ M.Stanitzki ${ }^{18}$, K.Stevenson ${ }^{36}$, A.Stocchi ${ }^{20}$, J.Strauss ${ }^{52}$, R.Strub ${ }^{10}$, B.Stugu ${ }^{4}$, M.Szczekowski ${ }^{53}$, M.Szeptycka ${ }^{53}$, T.Tabarelli ${ }^{29}$, A.Taffard ${ }^{23}$, F.Tegenfeldt ${ }^{50}$, F.Terranova ${ }^{29}$, J.Thomas ${ }^{36}$, J.Timmermans ${ }^{32}$, N.Tinti ${ }^{5}$, L.G.Tkatchev ${ }^{17}$, M.Tobin ${ }^{23}$, S.Todorova ${ }^{10}$, A.Tomaradze ${ }^{2}$, B.Tome ${ }^{22}$, A.Tonazzo ${ }^{9}$, L.Tortora ${ }^{40}$, P.Tortosa ${ }^{51}$, G.Transtromer ${ }^{25}$, D.Treille ${ }^{9}$, G.Tristram ${ }^{8}$, M.Trochimczuk ${ }^{53}$, C.Troncon ${ }^{28}$, A.Tsirou ${ }^{9}$, M-L.Turluer ${ }^{41}$, I.A.Tyapkin ${ }^{17}$, S.Tzamarias ${ }^{12}$, O.Ullaland ${ }^{9}$, 
V.Uvarov ${ }^{44}$, G.Valenti ${ }^{5}$, E.Vallazza ${ }^{48}$, C.Vander Velde ${ }^{2}$, P.Van Dam ${ }^{32}$, W.Van Den Boeck ${ }^{2}$, W.K.Van Doninck ${ }^{2}$, J.Van Eldik $^{32}$, A.Van Lysebetten ${ }^{2}$, N.Van Remortel ${ }^{2}$, I.Van Vulpen ${ }^{32}$, G.Vegni ${ }^{28}$, L.Ventura ${ }^{37}$, W.Venus ${ }^{38,9}$, F.Verbeure $^{2}$, M.Verlato ${ }^{37}$, L.S.Vertogradov ${ }^{17}$, V.Verzi ${ }^{39}$, D.Vilanova ${ }^{41}$, L.Vitale ${ }^{48}$, E.Vlasov ${ }^{44}$, A.S.Vodopyanov ${ }^{17}$, G.Voulgaris $^{3}, \quad$ V.Vrba ${ }^{13}, \quad$ H.Wahlen ${ }^{54}$, C.Walck ${ }^{46}$, A.J.Washbrook ${ }^{23}$, C.Weiser ${ }^{9}, \quad$ D.Wicke ${ }^{54}$, J.H.Wickens ${ }^{2}$, G.R.Wilkinson ${ }^{9}$, M.Winter ${ }^{10}$, M.Witek $^{19}$, G.Wolf ${ }^{9}$, J.Yi ${ }^{1}$, O.Yushchenko ${ }^{44}$, A.Zaitsev ${ }^{44}$, A.Zalewska ${ }^{19}$, P.Zalewski ${ }^{53}$, D.Zavrtanik ${ }^{45}$, E.Zevgolatakos ${ }^{12}$, N.I.Zimin ${ }^{17,25}$, A.Zintchenko ${ }^{17}$, G.C.Zucchelli ${ }^{46}$, G.Zumerle ${ }^{37}$

\footnotetext{
${ }^{1}$ Department of Physics and Astronomy, Iowa State University, Ames IA 50011-3160, USA
}

${ }^{2}$ Physics Department, Univ. Instelling Antwerpen, Universiteitsplein 1, BE-2610 Wilrijk, Belgium and IIHE, ULB-VUB, Pleinlaan 2, BE-1050 Brussels, Belgium

and Faculté des Sciences, Univ. de l'Etat Mons, Av. Maistriau 19, BE-7000 Mons, Belgium

${ }^{3}$ Physics Laboratory, University of Athens, Solonos Str. 104, GR-10680 Athens, Greece

${ }^{4}$ Department of Physics, University of Bergen. Allégaten 55, NO-5007 Bergen, Norway

${ }^{5}$ Dipartimento di Fisica, Università di Bologna and INFN, Via Irnerio 46, IT-40126 Bologna, Italy

${ }^{6}$ Centro Brasileiro de Pesquisas Físicas, rua Xavier Sigaud 150, BR-22290 Rio de Janeiro, Brazil

and Depto. de Física, Pont. Univ. Católica, C.P. 38071 BR-22453 Rio de Janeiro, Brazil

and Inst. de Física, Univ. Estadual do Rio de Janeiro, rua São Francisco Xavier 524, Rio de Janeiro, Brazil

${ }^{7}$ Comenius University, Faculty of Mathematics and Physics, Mlynska Dolina, SK-84215 Bratislava, Slovakia

${ }^{8}$ Collège de France, Lab. de Physique Corpusculaire, IN2P3-CNRS, FR-75231 Paris Cedex 05, France

${ }^{9}$ CERN, CH-1211 Geneva 23, Switzerland

${ }^{10}$ Institut de Recherches Subatomiques, IN2P3 - CNRS/ULP - BP20, FR-67037 Strasbourg Cedex, France

${ }^{11}$ DESY-Zeuthen, Platanenallee 6, D-15735 Zeuthen, Germany

${ }^{12}$ Institute of Nuclear Physics, N.C.S.R. Demokritos, P.O. Box 60228, GR-15310 Athens, Greece

${ }^{13}$ FZU, Inst. of Phys. of the C.A.S. High Energy Physics Division, Na Slovance 2, CZ-180 40, Praha 8 , Czech Republic

${ }^{14}$ Dipartimento di Fisica, Università di Genova and INFN, Via Dodecaneso 33, IT-16146 Genova, Italy

${ }^{15}$ Institut des Sciences Nucléaires, IN2P3-CNRS, Université de Grenoble 1, FR-38026 Grenoble Cedex, France

${ }^{16}$ Helsinki Institute of Physics, HIP, P.O. Box 9, FT-00014 Helsinki, Finland

17 Joint Institute for Nuclear Research, Dubna, Head Post Office, P.O. Box 79, RU-101 000 Moscow, Russian Federation

${ }^{18}$ Institut für Experimentelle Kernphysik, Universität Karlsruhe, Postfach 6980, DE-76128 Karlsruhe, Germany

${ }^{19}$ Institute of Nuclear Physics and University of Mining and Metalurgy, Ul. Kawiory 26a, PL-30055 Krakow, Poland

${ }^{20}$ Université de Paris-Sud, Lab. de l'Accélérateur Linéaire, IN2P3-CNRS, Bât. 200, FR-91405 Orsay Cedex, France

${ }^{21}$ School of Physics and Chemistry, University of Lancaster, Lancaster LA1 4YB, UK

${ }^{22}$ LIP, IST, FCUL - Av. Elias Garcia, 14-1 ${ }^{\circ}$, PT-1000 Lisboa Codex, Portugal

${ }^{23}$ Department of Physics, University of Liverpool, P.O. Box 147, Liverpool L69 3BX, UK

${ }^{24}$ LPNHE, IN2P3-CNRS, Univ. Paris VI et VII, Tour 33 (RdC), 4 place Jussieu, FR-75252 Paris Cedex 05, France

${ }^{25}$ Department of Physics, University of Lund, Sölvegatan 14, SE-223 63 Lund, Sweden

${ }^{26}$ Université Claude Bernard de Lyon, IPNL, IN2P3-CNRS, FR-69622 Villeurbanne Cedex, France

${ }^{27}$ Univ. d'Aix - Marseille II - CPP, IN2P3-CNRS, FR-13288 Marseille Cedex 09, France

${ }^{28}$ Dipartimento di Fisica, Università di Milano and INFN, Via Celoria 16, IT-20133 Milan, Italy

${ }^{29}$ Università degli Studi di Milano - Bicocca, Via Emanuelli 15, IT-20126 Milan, Italy

${ }^{30}$ Niels Bohr Institute, Blegdamsvej 17, DK-2100 Copenhagen $\varnothing$, Denmark

${ }^{31}$ IPNP of MFF, Charles Univ., Areal MFF, V Holesovickach 2, CZ-180 00, Praha 8, Czech Republic

32 NIKHEF, Postbus 41882, NL-1009 DB Amsterdam, The Netherlands

${ }^{33}$ National Technical University, Physics Department, Zografou Campus, GR-15773 Athens, Greece

${ }^{34}$ Physics Department, University of Oslo, Blindern, NO-1000 Oslo 3, Norway

${ }^{35}$ Dpto. Fisica, Univ. Oviedo, Avda. Calvo Sotelo s/n, ES-33007 Oviedo, Spain

${ }^{36}$ Department of Physics, University of Oxford, Keble Road, Oxford OX1 3RH, UK

${ }^{37}$ Dipartimento di Fisica, Università di Padova and INFN, Via Marzolo 8, IT-35131 Padua, Italy

${ }^{38}$ Rutherford Appleton Laboratory, Chilton, Didcot OX11 OQX, UK

${ }^{39}$ Dipartimento di Fisica, Università di Roma II and INFN, Tor Vergata, IT-00173 Rome, Italy

${ }^{40}$ Dipartimento di Fisica, Università di Roma III and INFN, Via della Vasca Navale 84, IT-00146 Rome, Italy

${ }^{41}$ DAPNIA/Service de Physique des Particules, CEA-Saclay, FR-91191 Gif-sur-Yvette Cedex, France

${ }^{42}$ Instituto de Fisica de Cantabria (CSIC-UC), Avda. los Castros s/n, ES-39006 Santander, Spain

${ }^{43}$ Dipartimento di Fisica, Università degli Studi di Roma La Sapienza, Piazzale Aldo Moro 2, IT-00185 Rome, Italy

${ }^{44}$ Inst. for High Energy Physics, Serpukov P.O. Box 35, Protvino, (Moscow Region), Russian Federation

${ }^{45}$ J. Stefan Institute, Jamova 39, SI-1000 Ljubljana, Slovenia and Laboratory for Astroparticle Physics,

Nova Gorica Polytechnic, Kostanjeviska 16a, SI-5000 Nova Gorica, Slovenia, and Department of Physics, University of Ljubljana, SI-1000 Ljubljana, Slovenia

${ }^{46}$ Fysikum, Stockholm University, Box 6730, SE-113 85 Stockholm, Sweden

${ }^{47}$ Dipartimento di Fisica Sperimentale, Università di Torino and INFN, Via P. Giuria 1, IT-10125 Turin, Italy

${ }^{48}$ Dipartimento di Fisica, Università di Trieste and INFN, Via A. Valerio 2, IT-34127 Trieste, Italy and Istituto di Fisica, Università di Udine, IT-33100 Udine, Italy

${ }^{49}$ Univ. Federal do Rio de Janeiro, C.P. 68528 Cidade Univ., Ilha do Fundão BR-21945-970 Rio de Janeiro, Brazil

${ }^{50}$ Department of Radiation Sciences, University of Uppsala, P.O. Box 535, SE-751 21 Uppsala, Sweden

${ }^{51}$ IFIC, Valencia-CSIC, and D.F.A.M.N., U. de Valencia, Avda. Dr. Moliner 50, ES-46100 Burjassot (Valencia), Spain

${ }_{52}$ Institut für Hochenergiephysik, Österr. Akad. d. Wissensch., Nikolsdorfergasse 18, AT-1050 Vienna, Austria

53 Inst. Nuclear Studies and University of Warsaw, Ul. Hoza 69, PL-00681 Warsaw, Poland

${ }^{54}$ Fachbereich Physik, University of Wuppertal, Postfach 100 127, DE-42097 Wuppertal, Germany 


\section{Introduction}

Supersymmetry (SUSY) may be broken at a scale below the grand-unification scale $M_{G U T}$, with the ordinary gauge interaction acting as the messenger of supersymmetry breaking $[1,2]$. In the corresponding models, the gravitino $\tilde{G}$ turns out to be the lightest supersymmetric particle (LSP) and is expected to be almost massless. The next-to-lightest supersymmetric particle (NLSP) is therefore unstable and decays, under the assumption of $R$-parity conservation, into an invisible gravitino and its ordinary matter partner.

The number of generations of supersymmetry breaking messengers in minimal models and the value of $\tan \beta$, usually determine which particle is the NLSP [3-6]. In this letter, lightest chargino pair production is searched for under the assumption that the $\tilde{\tau}_{1}$, the lightest of the mass eigenstates produced by the mixing of $\tilde{\tau}_{R}$ and $\tilde{\tau}_{L}$ [7], is the NLSP. If kinematically allowed, charginos could be produced at LEP through the exchange of a photon or a $Z$ in the s-channel, or through the exchange of an electron sneutrino in the $t$-channel. Each chargino would then promptly decay into a $\tilde{\tau}_{1}$ and a tau neutrino:

$$
e^{+} e^{-} \rightarrow \tilde{\chi}_{1}^{+} \tilde{\chi}_{1}^{-} \rightarrow \tilde{\tau}_{1}^{+} \tilde{\tau}_{1}^{-} \nu_{\tau} \bar{\nu}_{\tau}
$$

Being the NLSP, $\tilde{\tau}_{1}$ is expected to decay into $\tau+\tilde{G}$ with, in the laboratory frame, a mean decay length of:

$$
L=1.76 \times 10^{-3} \sqrt{\frac{E^{2}}{m_{\tilde{\tau}_{1}}^{2}}-1}\left(\frac{m_{\tilde{\tau}_{1}}}{100 \mathrm{GeV} / c^{2}}\right)^{-5}\left(\frac{m_{\tilde{G}}}{1 \mathrm{eV} / c^{2}}\right)^{2} \mathrm{~cm},
$$

which depends strongly on $m_{\tilde{\tau}}, m_{\tilde{G}}$ and the energy of the $\tilde{\tau}_{1}, E$.

It can be deduced from eq. (2) that for a given stau mass, different ranges of gravitino masses could determine three different final state topologies. For $m_{\tilde{G}} \lesssim 10 \mathrm{eV} / \mathrm{c}^{2}$ the stau decays at the interaction point. Thus, if chargino pairs were produced in this context, the final state topology would correspond to two acoplanar taus and missing energy and momentum. This signature has already been studied by the DELPHI Collaboration in the so-called leptonic channel of the search for charginos within the Minimal Supersymmetric Standard Model (MSSM) [8].

If the mass of the gravitino lay between 10 and $200 \mathrm{eV} / \mathrm{c}^{2}$, the staus would decay inside the tracking devices of the detector. Thus, the signature of chargino pair production would contain one or two tracks with either a kink or a decay vertex. In the case in which a stau would decay before reaching the active tracking volume, at least one track with a large impact parameter would be observed. This final state topology is almost identical to the one which would be produced by a pair of staus for the same range of gravitino masses, and has been studied by the in ref. [9].

Finally, if the mass of the gravitino is above $200 \mathrm{eV} / \mathrm{c}^{2}$, the staus would tend to decay outside the tracking devices, and would appear to be stable particles. A pair of tracks with anomalous ionization and Cerenkov radiation would then be observed. Also this final signature has been already studied by the DELPHI Collaboration in the search for stable heavy particles [10].

Thus, this letter is intended to make use without modification of the analyses already applied in the three searches described in refs. [8], [9] and [10]. Real data and simulated SM samples at $\sqrt{s} \sim 183 \mathrm{GeV}$ have already been analysed in this context, and the comparison between real and simulated data is included in refs. [8-10]. No excess over the expected SM background was observed. 
The selections developed for these three searches were thus applied to simulated data samples with different gravitino masses, and the results were interpreted in terms of $95 \%$ confidence level (CL) excluded regions in the $\left(m_{\tilde{\tau}_{1}}, m_{\tilde{\chi}_{1}^{+}}\right)$plane.

Section 2 describes the samples used for this work and presents the experimental results. Section 3 describes the interpretation of the results within one model with a gravitino LSP [4].

\section{Event sample and results}

The search is based on data collected by the DELPHI collaboration during 1997 at a centre-of-mass energy around $183 \mathrm{GeV}$. The total integrated luminosity was $53.9 \mathrm{pb}^{-1}$. A detailed description of the detector can be found in [11] and its performance in [12].

The program SUSYGEN [13] was used to generate the chargino pair production and decay. In order to compute detection efficiencies, a total of 45 samples of 500 events each were generated with gravitino masses of 1,100 and $1000 \mathrm{eV} / c^{2}, m_{\tilde{\tau}_{1}}+0.3 \mathrm{GeV} / c^{2} \leq$ $m_{\tilde{\chi}_{1}^{+}} \leq \sqrt{s} / 2$ and $m_{\tilde{\tau}_{1}} \leq 68 \mathrm{GeV} / c^{2}$. Samples with smaller $\Delta m=m_{\tilde{\chi}_{1}^{+}}-m_{\tilde{\tau}_{1}}$ were not generated because in that region each chargino decays into a $W$ and a gravitino with an appreciable branching ratio. In the aforementioned samples, charginos decay exclusively into a stau and a tau neutrino. The different background samples and event selections are described in references [8-10].

The generated signal and background events were passed through the detailed simulation [12] of the DELPHI detector and then processed with the same reconstruction and analysis programs used for real data. Table 1 shows the range of efficiencies, the main components and the total amount of the expected background events, and the number of observed data events for each sample ${ }^{1}$. Figures 1 and 2 show the distributions of some of the main variables used in the analyses described respectively in [8] and [9] for real data, expected standard model background, and a simulated signal of $m_{\tilde{\chi}_{1}^{+}}=85 \mathrm{GeV} / c^{2}$ and $m_{\tilde{\tau}_{1}^{+}}=69 \mathrm{GeV} / c^{2}$.

\begin{tabular}{|c||c|c|c|c|}
\hline Sample & Efficiencies (\%) & Main backgrounds & Expected b.g. & Observed events \\
\hline \hline$m_{\tilde{G}}=1 \mathrm{eV} / c^{2}$ & $24-35$ & $W W, \gamma \gamma$ & $10.7 \pm 1.3$ & 8 \\
\hline$m_{\tilde{G}}=100 \mathrm{eV} / c^{2}$ & $28-45$ & $\gamma \gamma$ & $0.63 \pm 0.55$ & 0 \\
\hline$m_{\tilde{G}}=1000 \mathrm{eV} / c^{2}$ & $25-63$ & $\mu \mu(\gamma)$ & $0.7 \pm 0.3$ & 0 \\
\hline
\end{tabular}

Table 1: Range of efficiencies, main sources of background, expected background and observed data events for the different analyses.

\section{Interpretation}

Since no evidence for a signal was found in the data, a limit on the production crosssection for chargino pairs was derived for each $\left(m_{\tilde{G}}, m_{\tilde{\tau}_{1}}, m_{\tilde{\chi}_{1}^{+}}\right)$combination. Figure 3 shows the $95 \%$ CL upper limit on the chargino pair production cross-section at $\sqrt{s}=183 \mathrm{GeV}$ as a function of $m_{\tilde{\chi}_{1}^{+}}$and $m_{\tilde{\tau}_{1}}$ for $\Delta m \geq 0.3 \mathrm{GeV} / c^{2}$ and $m_{\tilde{G}}=1,100$ and $1000 \mathrm{eV} / c^{2}$. These

\footnotetext{
${ }^{1}$ In the case of $m_{\tilde{G}}=1 \mathrm{eV} / c^{2}$, the expected background and observed events correspond to the sum of the degenerate and non-degenerate scenarios in the leptonic channels of [8], with errors added in quadrature.
} 
limits, which directly reflect the efficiencies of the applied selections, can be understood as follows:

$m_{\tilde{G}}=1 \mathrm{eV} / c^{2}: \quad$ When $m_{\tilde{\chi}_{1}^{+}}$and $m_{\tilde{\tau}_{1}}$ approach $m_{\mathrm{W}}$, the final state resembles that of $W$ pair production, and the efficiency becomes smaller. On the other hand, for small chargino masses the visible energy increases and the efficiency is affected by the requirement that some missing energy must be observed. In both cases, the 95\% CL limit on the chargino production cross section is increased.

$m_{\tilde{G}}=100 \mathrm{eV} / c^{2}: \quad$ The map of efficiencies is the result of the convolution of two factors. First, larger stau masses imply a smaller lifetime, and hence a smaller efficiency [9]. Secondly, a larger chargino mass leads to smaller stau momenta, and to smaller decay lengths.

$m_{\tilde{G}}=1000 \mathrm{eV} / \mathrm{c}^{2}: \quad$ In this case, the map of efficiencies is mainly affected by the momentum of the stau, because the method used to identify heavy stable particles relies on the lack of Cerenkov radiation in DELPHI's RICH detectors. To remove SM backgrounds, low momentum particles are removed, thus reducing the efficiency for higher chargino masses, especially in the region of small $\Delta m$.

Limits were derived in the frame of the model described in reference [4]. This general model assumes only radiatively broken electroweak symmetry and null trilinear couplings at the scale of the messengers' masses. The corresponding parameter space was scanned as follows: $1 \leq n \leq 4,5 \mathrm{TeV} \leq \Lambda \leq 900 \mathrm{TeV}, 1.1 \leq M / \Lambda \leq 9000,1.1 \leq \tan \beta \leq 50$, and $\mu>0$, where $n$ is the number of messenger generations in the model, $\Lambda$ is the ratio between the vacuum expectation values of the auxiliary component superfield and the scalar component of the superfield and $M$ is the messenger mass scale, tan $\beta$ and $\mu$ are defined as for the MSSM.

Figure 4 shows the regions excluded at $95 \%$ CL in the $\left(m_{\tilde{\chi}_{1}^{+}}, m_{\tilde{\tau}_{1}}\right)$ plane. The positiveslope area is excluded for all gravitino masses. The negative-slope area is excluded for $m_{\tilde{G}} \geq 100 \mathrm{eV} / c^{2}$. The area below $m_{\tilde{G}}=68.5 \mathrm{GeV} / c^{2}$ is excluded by the direct search for stau pair production [9]. The area of $\Delta m \leq 0.3 \mathrm{GeV} / c^{2}$ is not excluded because in this region the charginos do not decay mainly into $\tilde{\tau}_{1}$ and $\nu_{\tau}$ but on $\mathrm{W}$ and $\tilde{G}$. Therefore no events were generated in this region. Thus, if $\Delta m \geq 0.3 \mathrm{GeV} / c^{2}$, limits at $85.5,89.0$ and $89.0 \mathrm{GeV} / c^{2}$ can be set for $m_{\tilde{G}}=1,100$ and $1000 \mathrm{eV} / c^{2}$ respectively. The limit at $m_{\tilde{G}}=1 \mathrm{eV} / c^{2}$ is also valid for smaller masses of the gravitino, because they lead to the same final state topologies. The same argument is true for $m_{\tilde{G}} \geq 1 \mathrm{keV} / \mathrm{c}^{2}$. The chargino mass limit decreases with decreasing $m_{\tilde{\tau}_{1}}$ because in scenarios with gravitino LSP, small stau masses correspond to small sneutrino masses (both are proportional to $\Lambda$ ), and hence to smaller production cross-sections due to the destructive interference between the $s$ - and t-channels.

\section{Summary}

Lightest chargino pair production was searched for in the context of light gravitino scenarios. It was assumed that the $\tilde{\tau}_{1}$ is the NLSP. Three different searches were used in order to explore the $\left(m_{\tilde{\chi}_{1}^{+}}, m_{\tilde{\tau}_{1}}\right)$ plane in different domains of the gravitino mass.

The search in the context of very light gravitinos (the same as for MSSM chargino pair production with leptonic final states) produced eight candidate events to be compared to 
$10.7 \pm 1.3$ events expected from the SM background. An upper limit on the corresponding production cross-section between 0.36 and $0.48 \mathrm{pb}$ was derived at $95 \%$ CL.

The search in the context of medium range gravitino mass (leading to stau production with impact parameters or kinks), selected no events from the data, to be compared to $0.63 \pm 0.55$ events expected from the SM background. The $95 \%$ CL upper limit on the corresponding production cross-section varies between 0.13 and $0.23 \mathrm{pb}$.

The search in the context of heavier gravitinos (leading to the production of stable staus) gave no candidate events, with $0.7 \pm 0.3$ events expected from the SM background. An upper limit on the corresponding production cross-section between 0.10 and $0.35 \mathrm{pb}$ was set at $95 \%$ CL in the kinematically allowed region.

When combined, these results imply a $95 \%$ CL lower limit on the mass of the lightest chargino of $85.5 \mathrm{GeV} / c^{2}$, valid for $\Delta m \geq 0.3 \mathrm{GeV} / c^{2}$ and independently of the mass of the gravitino.

\section{Acknowledgements}

We are greatly indebted to our technical collaborators, to the members of the CERNSL Division for the excellent performance of the LEP collider, and to the funding agencies for their support in building and operating the DELPHI detector.

We acknowledge in particular the support of

Austrian Federal Ministry of Science and Traffics, GZ 616.364/2-III/2a/98,

FNRS-FWO, Belgium,

FINEP, CNPq, CAPES, FUJB and FAPERJ, Brazil,

Czech Ministry of Industry and Trade, GA CR 202/96/0450 and GA AVCR A1010521,

Danish Natural Research Council,

Commission of the European Communities (DG XII),

Direction des Sciences de la Matière, CEA, France,

Bundesministerium für Bildung, Wissenschaft, Forschung und Technologie, Germany,

General Secretariat for Research and Technology, Greece,

National Science Foundation (NWO) and Foundation for Research on Matter (FOM), The Netherlands,

Norwegian Research Council,

State Committee for Scientific Research, Poland, 2P03B06015, 2P03B1116 and SPUB/P03/178/98,

JNICT-Junta Nacional de Investigação Científica e Tecnológica, Portugal,

Vedecka grantova agentura MS SR, Slovakia, Nr. 95/5195/134,

Ministry of Science and Technology of the Republic of Slovenia,

CICYT, Spain, AEN96-1661 and AEN96-1681,

The Swedish Natural Science Research Council,

Particle Physics and Astronomy Research Council, UK,

Department of Energy, USA, DE-FG02-94ER40817. 


\section{References}

[1] M. Dine, W. Fischler and M. Srednicki, Nucl. Phys. B189 (1981) 575 ;

S. Dimopoulos and S. Raby, Nucl. Phys. B192 (1981) 353 ;

M. Dine and W. Fischler, Phys. Lett. B110 (1982) 227 ;

M. Dine and M. Srednicki, Nucl. Phys. B202 (1982) 238 ;

L. Alvarez-Gaumé, M. Claudson and M. Wise, Nucl. Phys. B207 (1982) 96 ;

C. Nappi and B. Ovrut, Phys. Lett. B113 (1982) 175 .

[2] M. Dine and W. Fischler, Nucl. Phys. B204 (1982) 346 ;

S. Dimopoulos and S. Raby, Nucl. Phys. B219 (1983) 479.

[3] J. A. Bagger, K. Matchev, D. M. Pierce and R. Zhang, Phys. Rev. D55 (1997) 3188.

[4] D. A. Dicus, B. Dutta, S. Nandi, Phys. Rev. D56 (1997) 5748 ;

D. A. Dicus, B. Dutta, S. Nandi, Phys. Rev. Lett. 78 (1997) 3055 ;

K. Cheung, D. A. Dicus, B. Dutta, S. Nandi, Phys. Rev. D58 (1998) 57705 .

[5] F. Borzumati, On the Minimal Messenger Model, hep-ph/9702307 and WIS/96-50PH, Dec. 1996.

[6] G. F. Giudice, R. Rattazzi, Theories with Gauge-Mediated Supersymmetry Breaking, CERN-TH/97-380, Jan. 1998 and hep-ph/9801271. Submitted to Phys. Rev. D.

[7] A. Bartl et. al., Z. Phys. C73 (1997) 469.

[8] DELPHI Collaboration, P. Abreu et al., Phys. Lett. B446 (1999) 75.

[9] DELPHI Collaboration, P. Abreu et al., E. Phys.J. C7 (1999) 595.

[10] DELPHI Collaboration, P. Abreu et al., Phys. Lett. B444 (1998) 491.

[11] DELPHI Collaboration, P. Aarnio et al., Nucl. Instr. and Meth. 303 (1991) 233.

[12] DELPHI Collaboration, P. Abreu et al., Nucl. Instr. and Meth. 378 (1996) 57.

[13] SUSYGEN 2.20, S. Katsanevas and S. Melachroinos in Physics at LEPD, CERN 9601, Vol. 2, p. 328 and http://lyoinfo.in2p3.fr/susygen/susygen.html ;

S. Katsanevas and P. Moravitz, Comp. Phys. Com. 122 (1998) 227. 

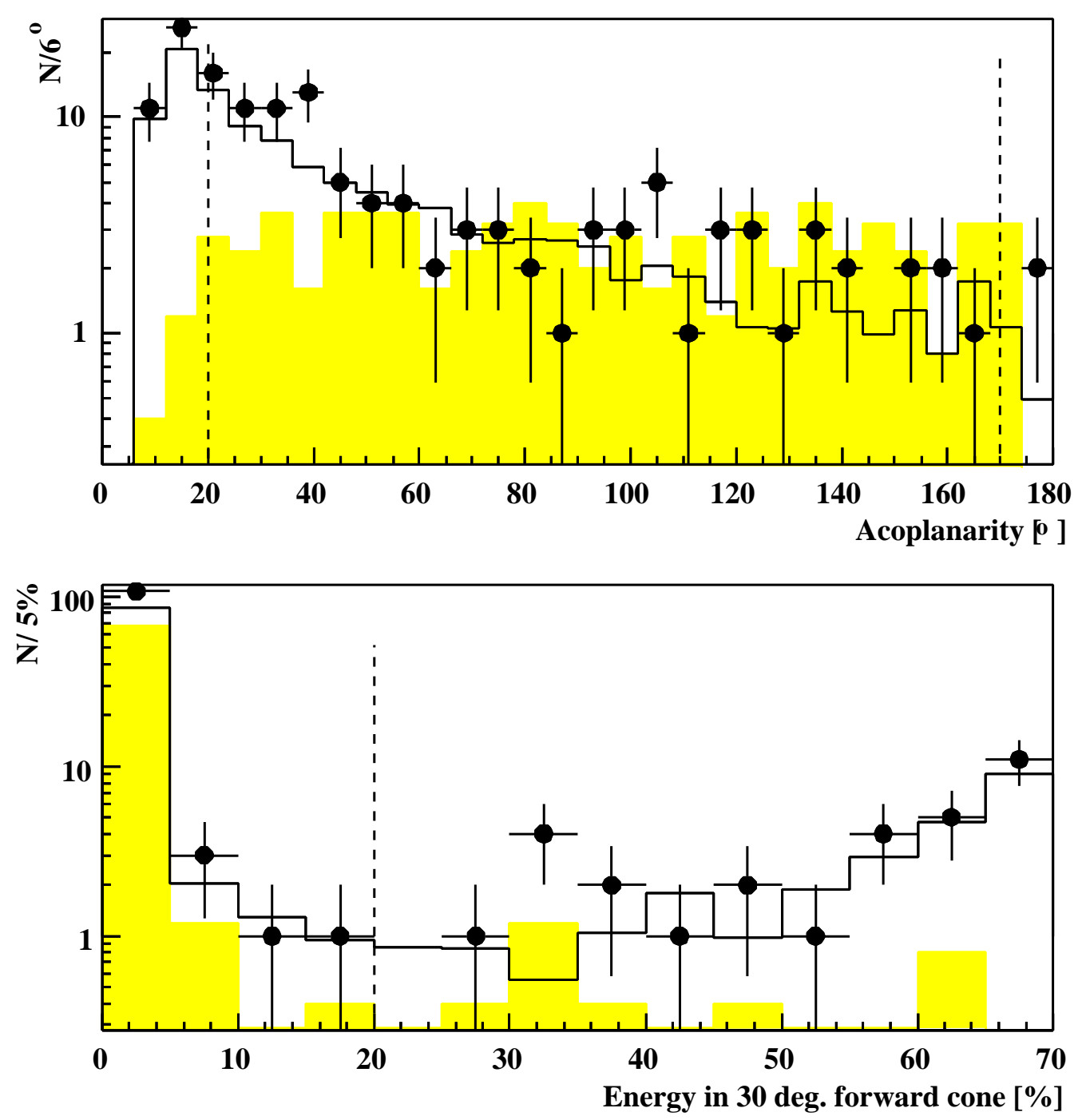

Figure 1: Coplanarity between the two lepton tracks (above) and percentage of energy deposited in a $30^{\circ}$ forward cone (below), for real data (dots), expected Standard Model background (blank histogram) and simulated signal for $m_{\tilde{\chi}_{1}^{+}}=85 \mathrm{GeV} / c^{2}$ and $m_{\tilde{\tau}_{1}^{+}}=69 \mathrm{GeV} / c^{2}$ decaying with a mean distance of $50 \mathrm{~cm}$ (dark histogram). The segmented lines indicate selection criteria imposed as explained in [8]. 

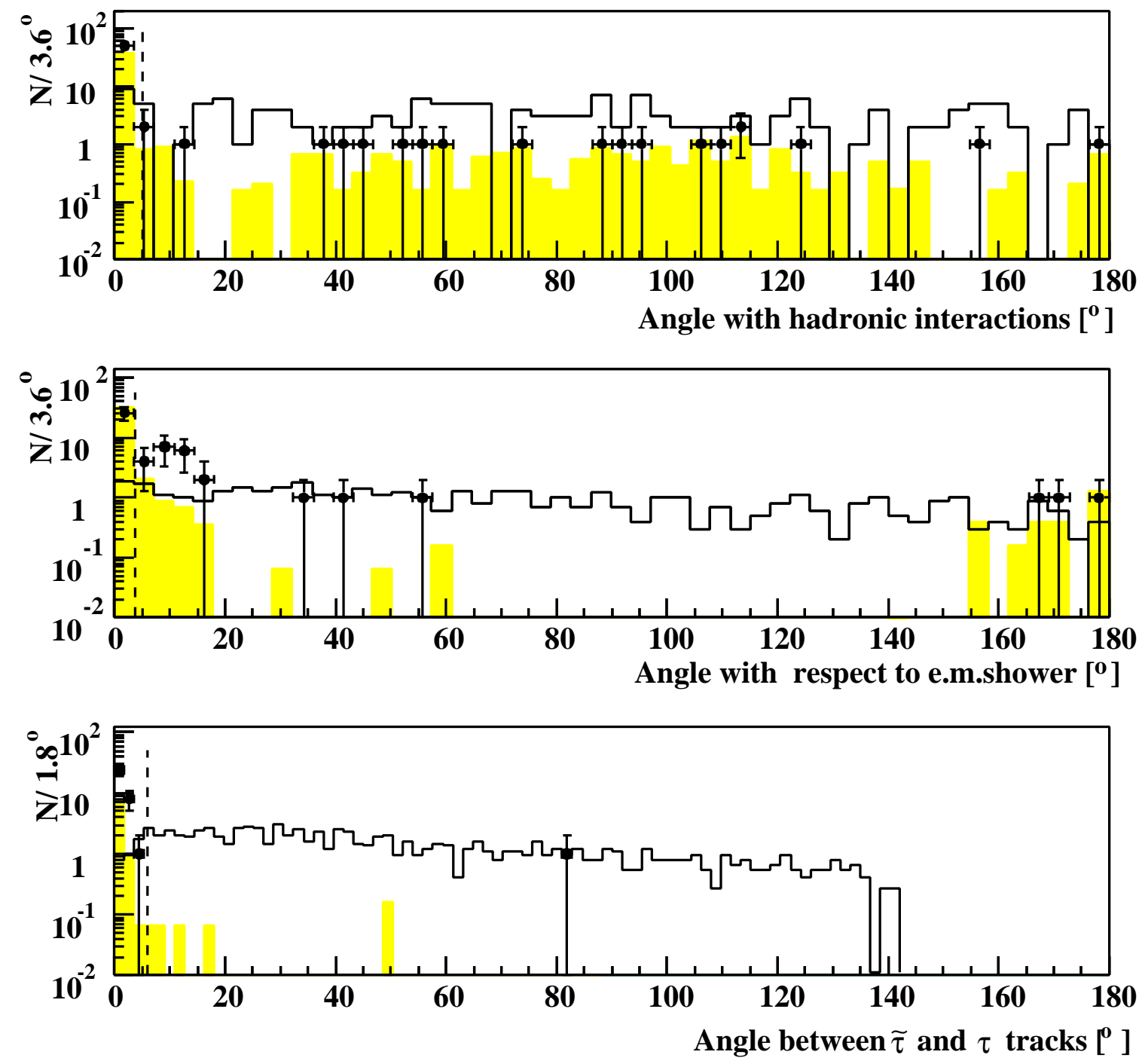

Figure 2: Angle between the hadronic interaction (if any) and the reconstructed vertex (above), angle between the electromagnetic shower and the direction defined by the $\mathrm{d}-$ ifference between the momenta of $\tilde{\tau}_{1}$ and its associated $\tau$, defined at the crossing point (middle), and angle between the tracks of the kink (below), for real data (dots), expected Standard Model background (dark histogram) and simulated signal for $m_{\tilde{\chi}_{1}^{+}}=85 \mathrm{GeV} / \mathrm{c}^{2}$ and $m_{\tilde{\tau}_{1}^{+}}=69 \mathrm{GeV} / c^{2}$ decaying with a mean distance of $50 \mathrm{~cm}$ (blank histogram). The segmented lines indicate selection criteria imposed as explained in [9]. 

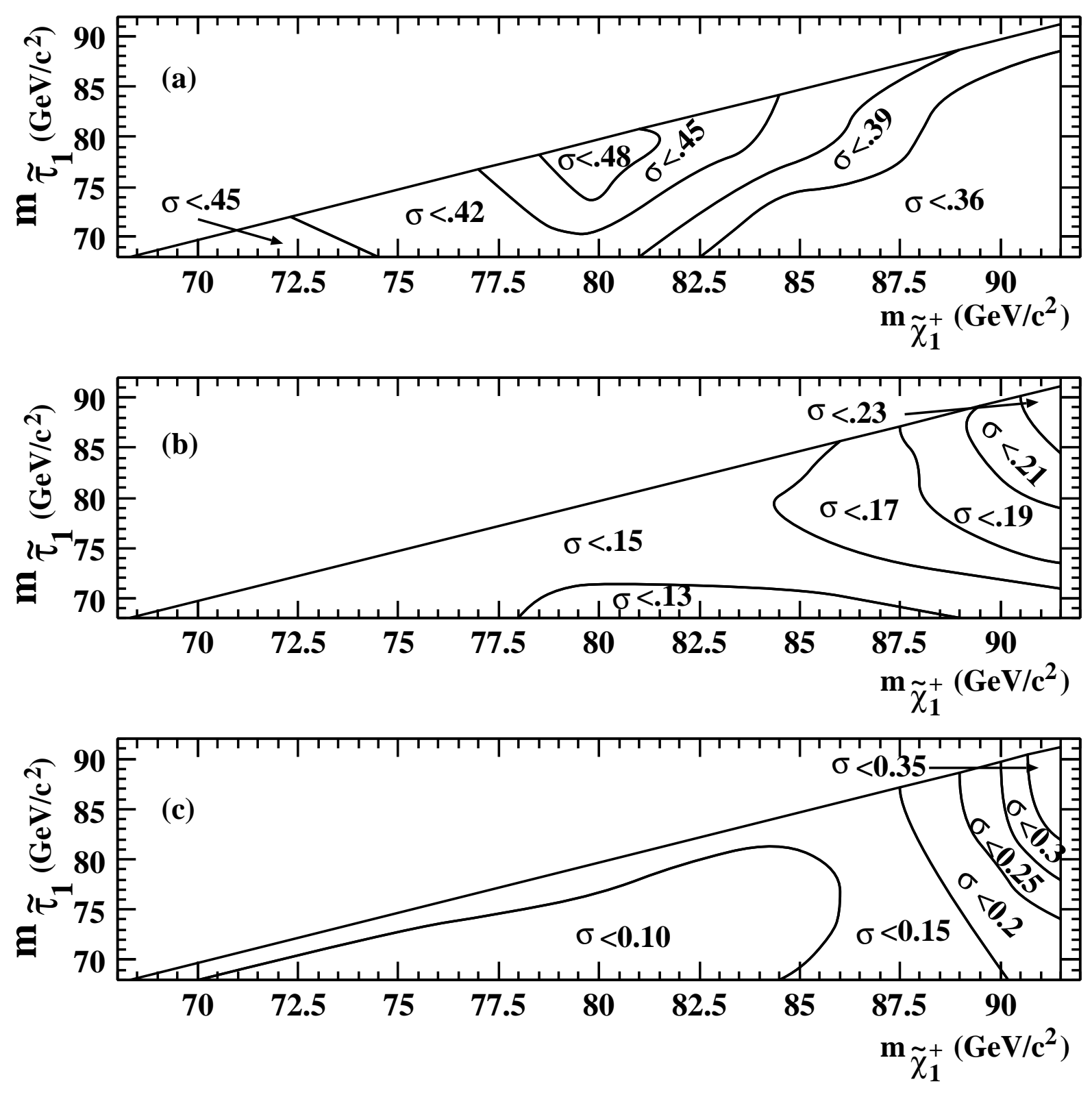

Figure 3: Limits in picobarn on lightest chargino pair production cross-section at $95 \% \mathrm{CL}$. Limits are shown as functions of $m_{\tilde{\chi}_{1}^{+}}$and $m_{\tilde{\tau}_{1}}$ for (a) $m_{\tilde{G}}=1 \mathrm{eV} / \mathrm{c}^{2}$, (b) $m_{\tilde{G}}=100 \mathrm{eV} / \mathrm{c}^{2}$ and (c) $m_{\tilde{G}}=1000 \mathrm{eV} / \mathrm{c}^{2}$. 


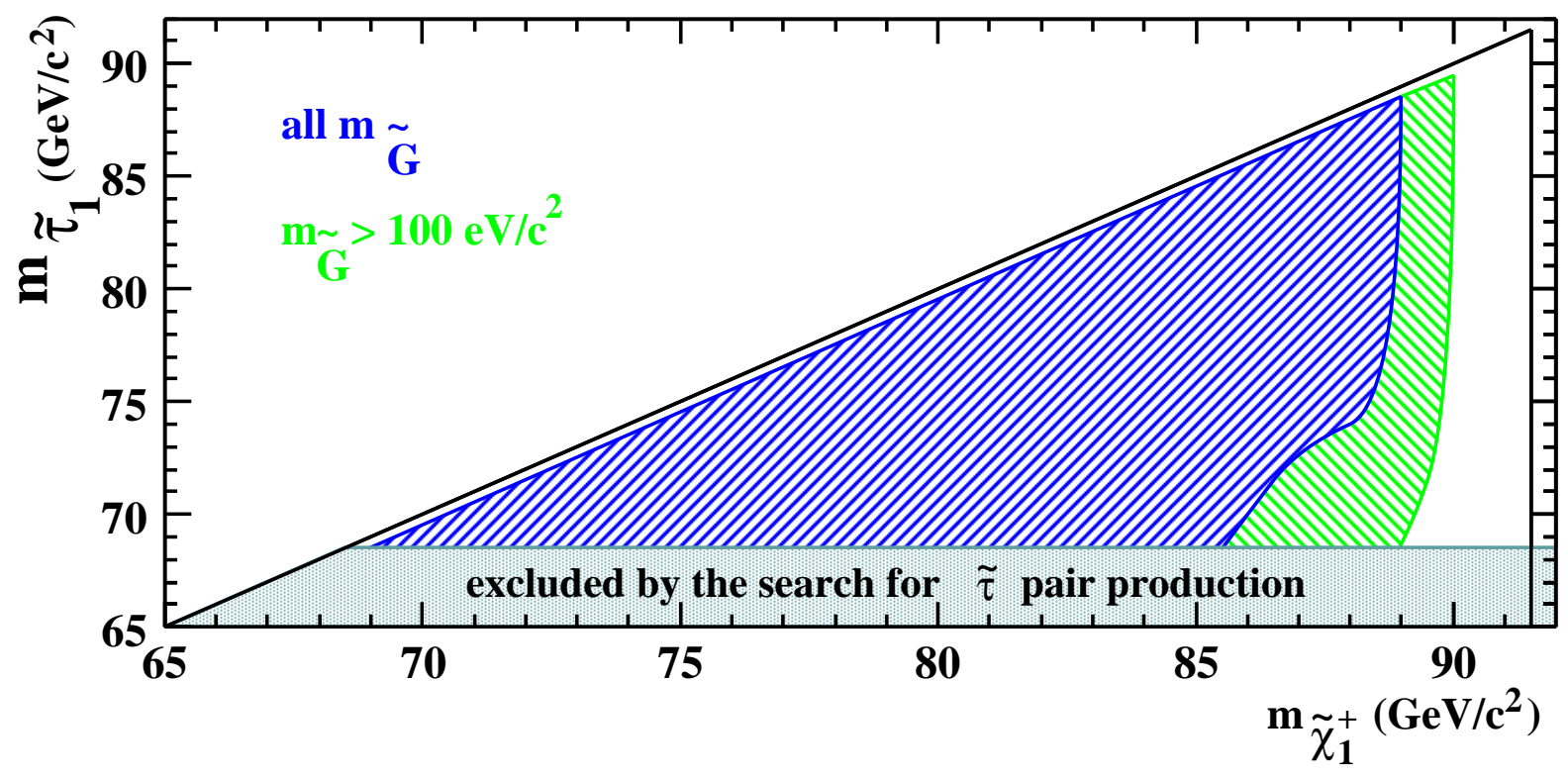

Figure 4: Areas excluded at 95\% CL in the $\left(m_{\tilde{\chi}_{1}^{+}}, m_{\tilde{\tau}_{1}}\right)$ plane. The positive-slope area is excluded for all $m_{\tilde{G}}$. The negative-slope area is excluded for $m_{\tilde{G}} \geq 100 \mathrm{eV} / \mathrm{c}^{2}$. The grey area is excluded by the direct search for stau pair production. 